\title{
DILUTED MAGNETIC SEMICONDUCTOR INVESTIGATIONS IN UKRAINE. NATURE OF SOME ADDITIONAL LINES IN QW OPTICAL SPECTRA
}

\author{
S.M. Ryabchenko*, V.G. Abramishvili, A.V. Komarov \\ Institute of Physics of National Academy of Sciences of Ukraine \\ 46, Prospekt Nauki st., Kiev, 252028, Ukraine \\ Yu.G. Semenov ${ }^{\dagger}$, F.V. KYRYCHenko
}

Institute of Semiconductor Physics of National Academy of Sciences of Ukraine 45, Prospekt Nauki st., Kiev, 252028, Ukraine

AND J.J. DuBowskI

Institute for Microstructural Science of NRC of Canada

Ottawa, Ontario, Canada KlA OR6

The general review of the history and present-day situation of diluted magnetic semiconductor investigation in Ukraine is given by S. Ryabchenko. Some noteworthy results of diluted magnetic semiconductor investigation obtained in Ukraine are pointed out. The main features of the present day situation are mentioned also. As an example of last diluted magnetic semiconductor investigations, the new result obtained by Abramishvili, Komarov, Ryabchenko, Semenov, Kyrychenko and Dubowski for $\mathrm{Cd}_{1-x} \mathrm{Mn}_{x} \mathrm{Te} / \mathrm{CdTe} /$ $\mathrm{Cd}_{1-x} \mathrm{Mn}_{x}$ Te quantum well structures grown by laser ablation method are presented. A weak additional line was observed in the reflectivity spectra of a $27 \AA$ wide quantum well with $x=0.11$ in the barrier. Such additional line has not been observed in spectra of similar molecular beam epitaxy grown structures. Based on the theoretical computations of the energies and the relation of intensities of the main and additional lines we conclude that this line might be associated with hh2 $\rightarrow$ e1 transitions, which ceases to be forbidden in the presence of technologically caused asymmetry of quantum well potential profile.

PACS numbers: 78.55.Et

*e-mail address: ryabch@iop.kiev.ua

†e-mail address: max@spin.kiev.ua 


\section{Introduction}

In general, physics of diluted magnetic (or semimagnetic, or magnetically mixed) semiconductors (DMSs) presents an intersection of magnetic phenomena physics, semiconductor physics and physics of disordered solid solutions. From the magnetism point of view, the main peculiarities of DMS physics are connected with the carrier-ion exchange interaction. Of course, the problem of localized non-coupled electrons exchange interaction (ion-ion exchange interaction) in these materials is not out of interest, however, as a rule, this problem is closely linked to more detailed features of the material under consideration.

The problem of the carrier-ion exchange interaction in metal have been developed by Vonso vskij with co-workers [1-3], see also [4], Kondo [5] and others. One of the first considerations of the electron-magnetic impurity exchange interaction in the case of semiconductors has been performed by Pines, Bardin and Slichter [6] in 1957 in connection with the spin relaxation mechanism. In succeeding years a lot of experimental and theoretical investigations were carried out on the physics of magnetic semiconductors. It had been reviewed and generalized in the monographs of Metfessel and Mattis [7], and Nagaev [8]. The carrier-ion exchange interaction is of primary importance in magnetic semiconductors, but in most cases the ion-ion exchange interaction is a determining factor for characteristics of the materials. So, we have no possibilities to change easily these characteristics by an external influence.

It seems to be the main reason why the magnetic semiconductor and DMS investigations were developed independently in most cases. Finally, on the late of 70s a lot of DMS effects turn out to be unexpected. The DMSs have not been treated as a special class of materials.

As a result of this situation, most researches, which gave impetus to DMS investigations, were started as investigations of semiconductors with magnetic impurities. Firstly in Ukraine such researches were started in the Institute of Semiconductors of Academy of Sciences of Ukraine (IS ASU) in Kiev under leadership of Dejgen, who was the founder of the department of Radiospectroscopy of Semiconductors in the Institute and the originator of the "Kiev's School of Radiospectroscopy". In 1975 Dejgen et al. [9] paid attention to the carrier-ions exchange interaction problem in semiconductors in connection with the spin relaxation problem of paramagnetic centers. In late of 60 s the magneto-optical and optically detected magnetic resonance (ODMR) investigations of magnetic centers in dielectric and semiconductor crystals were started in the Institute of Physics of Academy of Sciences of Ukraine (IP ASU) in Kiev.

\section{DMS investigations in Ukraine}

The DMS (in present day terms) investigations in Ukraine were started in early 70s from the fabrication of II-VI semiconductor solid solutions with cations partially substituted by $3 d$ magnetic ions. This work has been performed in Chernivtsy State University and IS ASU. The investigations of the optical and magnetic properties of these materials in IP ASU resulted in the first observation and explanation of exciton giant spin splitting (GSS) effect in terms of carrier-ion exchange 
interaction, $[10,11]$. Further, the intensive and fruitful investigations were performed at IP ASU (today named as lnstitute of Physics of National Academy of Sciences of Ukraine - IP NASU), IS ASU (today named as Institute of Physics of Semiconductors - IPS NASU), Chernivtsy University, Donetsk Physical and Technical Institute of NASU.

It is difficult to give a complete review of all these researches. They resulted in the demonstration of a new type of ODMR due to changing of GSS under $3 d$-ions EPR pumping conditions [11], prediction of ferromagnetic ordering in DMSs with a high carrier density [12], interpretation of the sign difference of exchange integrals for conductivity and valence band electrons [13], theory of magnetic polarons in DMSs with the exact solution for the statistical sum in the framework of "exchange box" model [14], theory and manifestation of exchange scattering effects [15], and many other phenomena.

A lot of applied investigations of narrow gap DMS growth technology for scientific purposes as well as for modern optical engineering devices have been performed in the Design and Technological Department of Chernivtsy University. Some of them were taken for commercial production. The high quality wide gap DMS bulk materials have been also growing in the Department.

A lot of significant results have been obtained in cooperation with scientists from Russia (St. Petersburg, Ekaterinburg, Tchernogolovka), Poland, France, Germany, Canada and other countries.

The present situation in Ukraine from the standpoint of scientific activity conditions is characterized by such factors as:

(i) General economical difficulties; dramatic decreasing in the industry interest to applied sciences and investigations; cancellation of high technological production.

(ii) Reduction of fundamental research funding from the state budget and cancellation of it from the industry side.

(iii) Shortening of working places for scientists and engineers; brain drain.

(iiii) Decreasing in flow of talented youth in science, especially in natural and technological sciences.

At the same time enhancement of international scientific cooperation with former Soviet Union countries is present. The old scientific contacts with scientists from Russia and other countries of Community of Independent States are conserved in the main part.

All centers of DMS investigations continue their activity. The topics of studies are coordinated with prominent tendencies in the world science. However, the level of some types of experiments is limited due to the absence of modern equipment for measurements or sample preparations.

For instance, in present days a lot of theoretical and experimental investigations of quantum structures (in the most part - quantum wells (QWs)) are performed by Ukrainian physicists. The DMS quantum structures are also widely investigated. Unfortunately, there are no modern sample growth laboratories in Ukraine now.

Ukrainian scientists now receive some international support from INTAS, CRDF grants, other bilateral and many-sided channels of international scien- 
tific cooperation. Certain parts of DMS investigations in Ukraine are funded by Ukrainian State Fundamental Research Foundation, by NASU and Ministry of Education of Ukraine.

Finally we present a new result obtained for DMS based $\mathrm{Cd}_{1-x} \mathrm{Mn}_{x} \mathrm{Te} / \mathrm{CdTe} /$ $\mathrm{Cd}_{1-x} \mathrm{Mn}_{x} \mathrm{Te} \mathrm{QW}$ structures, which makes a new way of looking at some optical lines in quantum well spectra.

\section{About the nature of some additional lines in QW spectra}

\subsection{Introduction to the problem}

In the last years the particular attention gives to consideration of the systems with reduced dimensionality, in particular, quasi two-dimensional systems or quantum wells. Such structures comprise of a thin layer of one material sandwiched between two layers of another material. Because of technology features the boundary between two different materials is not perfectly sharp. This has raised a question about the influence of non-ideal interface on the properties of quasi two-dimensional systems.

It is known (see, for instance, $[16,17])$ that the interface intermixing in the case of II-VI diluted magnetic semiconductor quantum wells leads to the paramagnetic enhancement effect in such structures. In this work we would like to point our attention to another effect connected with non-ideal interface profile. In the case of asymmetrical interface profile the real potential shape of quantum well becomes asymmetrical also. This reduces the symmetry of Hamiltonian and the eigenfunctions of one-dimensional problem in the growth direction are neither even nor odd functions further. This fact breaks the symmetry selection rules for optical interband transitions and makes possible to observe the optical transitions between "even" and "odd" confined states, which are strictly forbidden in the case of rectangular (or other symmetrical profile) quantum well.

Such additional lines have been observed in a number of samples grown by different techniques. In the relatively good quality samples grown by the MBE technique such additional lines are observed in very limited cases [18]. As it will be shown in this paper in the samples grown by the laser ablation technique, the intensity of the additional line can reach about $10 \%$ of the main line intensity. In the experiments performed in Ref. [18] for a $20 \AA$ wide $\mathrm{CdTe} / \mathrm{Cd}_{0.88} \mathrm{Mn}_{0.12} \mathrm{Te}$ quantum well the authors observed the additional line in reflectivity spectra in $\sigma^{+}$ polarization for magnetic field $H>2 \mathrm{~T}$. The observation of the additional line has been used as evidence of type I - type II transition in the magnetic field and associated with e1-hh3 transition. Such explanation of the results requested the valence band offset parameter in $\mathrm{CdTe} / \mathrm{CdMnTe}$ quantum well structures to be equal to $Q=0.1 \div 0.15$.

However, the numerous studies of $\mathrm{Cd}_{1-x} \mathrm{Mn}_{x} \mathrm{Te} / \mathrm{CdTe} / \mathrm{Cd}_{1-x} \mathrm{Mn}_{x} \mathrm{Te} \mathrm{QWs}$ show that the valence band offset parameter value is rather $0.3 \div 0.45$ than 0.15 . The measurements performed for half-parabolic QWs [19] provide good evidence of it. Due to asymmetry of half-parabolic potential profile it is possible to observe transitions between electron and hole confined states with the same values of $n$ and $m$ ( $n, m$ are numbers of the electron and hole confined states, respectively) as well as transitions between states, with different values $n$ and $m$. Namely, the 
analysis of the energy positions of the e1-hh1 and e1-hh2 and/or e2-hh1 optical transitions allowed in the case of asymmetrical potential profile gives the authors the possibility to determine the valence band offset parameter to be equal to $Q=0.4 \pm 0.05$.

In the same time the analysis of interface intermixing effect influence on e1-hh1 exciton giant spin splitting in QWs $[16,17]$ and studies of "normal" and "inverted" interfaces in $\mathrm{Cd}_{1-x} \mathrm{Mn}_{x} \mathrm{Te} / \mathrm{CdTe} / \mathrm{Cd}_{1-y} \mathrm{Mg}_{y} \mathrm{Te} \mathrm{QW}$ structures distinctly show that the potential profile of MBE grown "rectangular" QWs has a technologically caused asymmetry. So, our model of asymmetrical quantum wells allows us to explain the results obtained in Ref. [18] in terms of "forbidden" transitions e1-hh2 with a realistic value of the valence band offset parameter.

Note that since there are no excited electron and hole quantum confined states in the $20 \AA$ wide $\mathrm{Cd}_{0.88} \mathrm{Mn}_{0.12} \mathrm{Te} / \mathrm{CdTe} / \mathrm{Cd}_{0.88} \mathrm{Mn}_{0.12} \mathrm{Te} \mathrm{QW}$, for interpretation of the results of their experiment the authors of Ref. [18] used the model of "effective potential" to describe the heavy hole states with respect to the electron-hole Coulomb interaction influence. Indeed, there are two possible types of the excitons in QW. The first is the exciton associated with both electron and hole confined by the QW potential. The second type is the excitons comprised of electron (hole) confined by the QW potential and hole (electron) confined due to the Coulomb interaction only. In fact, the states of the second type excitons may be considered as additional (i.e. possible only if the Coulomb interaction is taken into account) quasi one-particle states hh2 (e2), hh3 (e3), etc.

Due to the technological features the samples grown by laser ablation method might have more asymmetrical interface profiles than the samples grown by the MBE technique. The additional line in spectra of such samples may be observed both in $\sigma^{+}$and $\sigma^{-}$polarizations of light and its intensity may be comparable with the main line. We will show that such situation is really present for our samples grown by the laser ablation method and we will describe it using the proposed model.

\subsection{Experiment}

The samples were prepared by the laser ablation method by one of us, J.J. Dubowski, Ottawa, Canada. One of them (CCM-205) contains 6 CdTe QWs separated by $\mathrm{Cd}_{0.89} \mathrm{Mn}_{0.11}$ Te barriers. The well and barrier widths are $L_{\mathrm{w}}=27 \AA$ (it was planned in the growth process to make $L_{\mathrm{w}}=20 \AA$ ) and $L_{\mathrm{b}}=165 \AA$, respectively. The second structure (CCM-207) consists of $41 \mathrm{CdTe} / \mathrm{Cd}_{0.89} \mathrm{Mn}_{0.11} \mathrm{Te} \mathrm{QWs}$ with the same value of $L_{\mathrm{w}}$ parameter, but the barrier width is reduced to $L_{\mathrm{b}}=45 \AA$ value. The barriers in CCM-207 are narrow enough for a creation of a miniband structure both for electron and heavy hole subsystems. So, this sample should be considered as a superlattice (SL) while the CCM-205 we consider as a multi-quantum well (MQW). A more detailed description of the samples parameters is given in Ref. [20]. The comparison of the experimental values of photoluminescence peak positions with those calculated for rectangular QW are performed in Ref. [20]. This comparison presents evidence of the SL effects in CCM-207 structure. The authors of Ref. [20] conclude also that $L_{\mathrm{w}}$ in the both structures is some greater than it was planned (up to $27 \AA$ instead of $20 \AA$ ). 


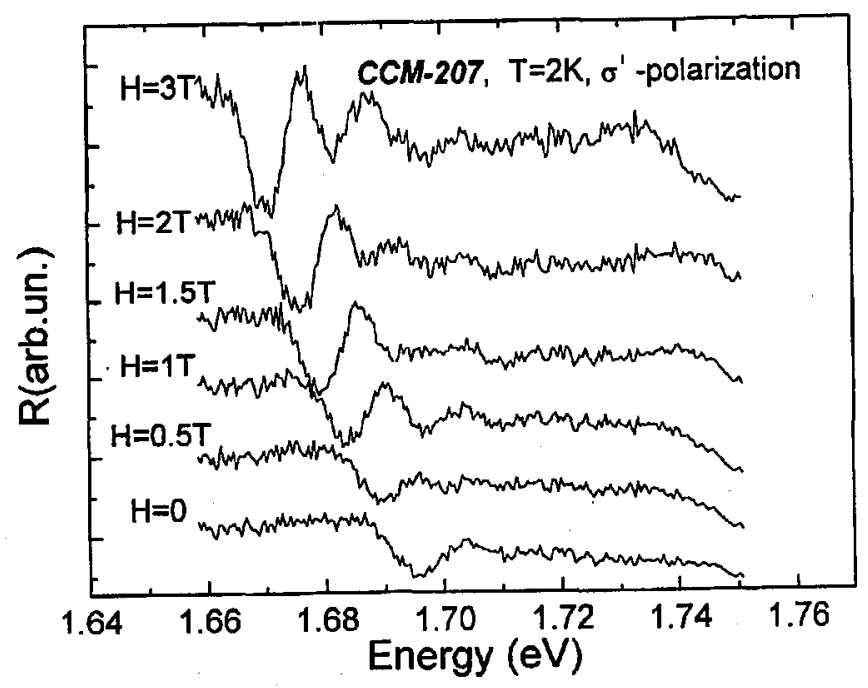

Fig. 1. The reflectivity spectra for CCM-207 sample in $\sigma^{+}$polarization for different values of the magnetic field $H$.
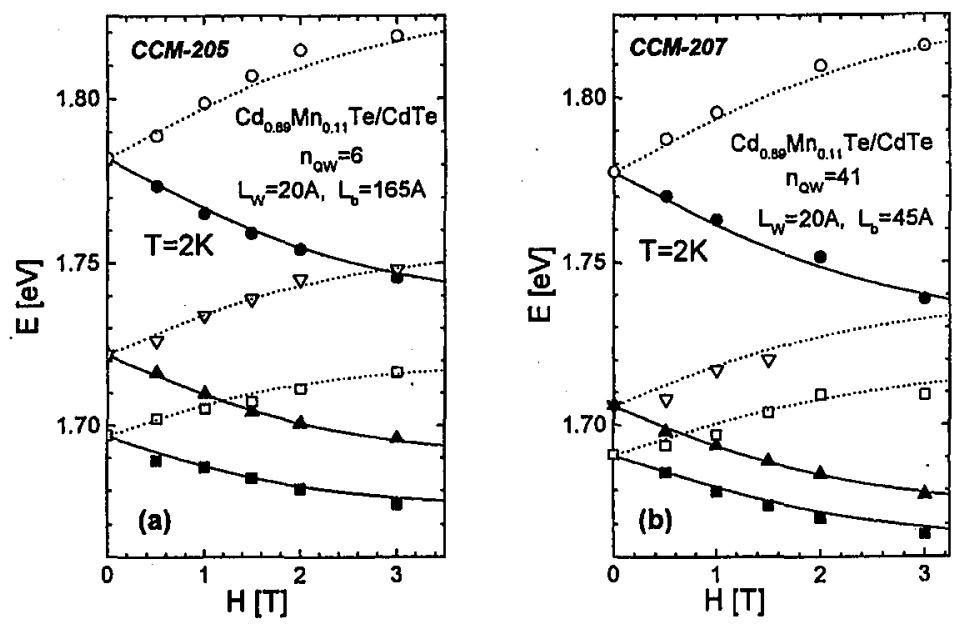

Fig. 2. Magnetic field dependence of the optical transition energies for CCM-205 (a) and CCM-207 (b) samples. The squares, triangles and circles correspond to the main line, additional line and barrier exciton, respectively. The experimental points are approximated by curves for convenience. Solid symbols and solid lines correspond to $\sigma^{+}$ polarization while open symbols and dotted lines to $\sigma^{-}$polarization.

The measurements were performed in a temperature of $T=2 \mathrm{~K}$. The additional line for both samples was observed in the reflectivity spectra. In Fig. 1 the reflectivity spectra of CCM-207 in $\sigma^{+}$polarization are shown for different values of the external magnetic field $H$. Each of these spectra includes the line associated 
with the barrier exciton, the main e1-hh1 line and the additional line blue shifted from the main one. The energy distance between the main and additional lines in the case of SL $(15 \pm 1 \mathrm{meV})$ is slightly less than in the MQW case $(24 \pm 2 \mathrm{meV})$. The additional line intensity increases a little with magnetic field in $\sigma^{+}$polarization and decreases significantly without a noticeable line broadening in $\sigma^{-}$.

In Fig. 2 we plot the magnetic field dependence of the optical transition energies for the samples CCM-205 (a) and CCM-207 (b). The additional line has no diamagnetic shift proportional to $\mathrm{H}^{2}$, so it cannot be treated as the excited e1-hh1 exciton state. We can see also for both samples that the GSS values of the additional line are some greater than those for the main line. This make us not to be able to describe the additional line as a light hole exciton. Moreover, this fact tells us that probably the heavy hole state associated with the additional optical transition is less confined than hh1 state associated with e1-hh1 exciton. As far as the additional line is observed both in $\sigma^{+}$and $\sigma^{-}$polarizations the approach developed in Ref. [18] and based on the presence of type I - type II transition in the magnetic field cannot be applied in this situation.

Since in the MBE grown structures the additional lines with comparable intensities have not been observed we suppose that the nature of the additional optical transitions is connected to the growth technique peculiarities. Due to the features of technological process used for preparation of the samples CCM-205 and CCM-207, these quantum structures may have a strong asymmetrical profile of the interfaces. As mentioned above, this allow us to consider the possibilities of the optical transitions forbidden in the case of symmetrical potential profile.

\subsection{Theoretical description}

We choose $z$-axis to be along the growth direction. Following Refs. $[16,17]$ we assume the Mn-impurity concentration in the structure to be a continuous function of the coordinate $x(z)$. We label $L_{\mathrm{w}}$ as the well width in the rectangular well approximation. Now we introduce the interface function $F\left(z-z_{\mathrm{if}}\right)$ which describes the realistic interface profile. This function takes the values 1 and 0 away from the interface point $z_{\text {if }}$ to the left side $\left(z \ll z_{\text {if }}\right)$ and to the right side $\left(z \gg z_{\text {if }}\right)$, respectively. The possible expressions for the function $F(t)$ will be discussed below. So, the real Mn-impurity concentration profile in $z$-direction for a single $\mathrm{CdTe} / \mathrm{Cd}_{1-x} \mathrm{Mn}_{x} \mathrm{Te}$ quantum well can be represented as the continuous function $x(z)$

$$
x(z)=x_{\mathrm{B}}\left[F(z-0)+1-F\left(z-L_{\mathrm{w}}\right)\right],
$$

where $z=0$ and $z=L_{\mathrm{w}}$ correspond to the interface positions in the case of rectangular QW approximation. The function $x(z)$ becomes asymmetrical if the function $\widetilde{F}(t)=F(t)-1 / 2$, where $t=z-z_{\mathrm{if}}$, is not the antisymmetrical function $(\widetilde{F}(-t) \neq-\widetilde{F}(t))$.

For our calculations we used two types of the interface functions $F(t)$. The first is a "one-stage" function, which can be written either in terms of power-functions

$$
F(t)= \begin{cases}1 & t<0 \\ {\left[1+\left(t / L_{\mathrm{D}}\right)^{s}\right]^{-1}} & t \geq 0\end{cases}
$$


or in terms of exponential functions

$$
F(t)=\left\{\begin{array}{ll}
1 & t<0 \\
\exp \left(-t / L_{\mathrm{D}}\right) & t \geq 0
\end{array} .\right.
$$

Here $L_{\mathrm{D}}$ is the characteristic interface mixing length, $s$ is a parameter. The interface function in the form (2) vanishes more slowly with increasing $t$ than that in the form (3) and provides more long tails in the Mn distribution function $x(z)$.

The second type of the interface functions under consideration is a "two-stage" interface function, where we assume the presence of two sources of Mn concentration changes during the growth process. Each of these mechanisms is characterised by its own parameter $L_{\mathrm{D}}$. The parameter $f$ describes the relative contribution of the faster process in the dynamics of the Mn-impurity changing

$$
F_{2}(t)= \begin{cases}1 & t<0 \\ f\left[1+\left(t / L_{\mathrm{D} 1}\right)^{s 1}\right]^{-1}+(1-f)\left[1+\left(t / L_{\mathrm{D} 2}\right)^{s 2}\right]^{-1} & t \geq 0\end{cases}
$$

or in terms of exponential functions

$$
F_{2}(t)= \begin{cases}1 & t<0 \\ f \exp \left(-t / L_{\mathrm{D} 1}\right)+(1-f) \exp \left(-t / L_{\mathrm{D} 2}\right) & t \geq 0\end{cases}
$$

For the calculation of the potential shape $U(z)$ in the magnetic field we used the method developed in Refs. $[16,17]$ assuming that the local magnetization at the point $z=z_{0}$ is equal to the magnetization of a bulk crystal with the same value of $\mathrm{Mn}$ concentration $x\left(z=z_{0}\right)$. Then we solved corresponding one-dimensional
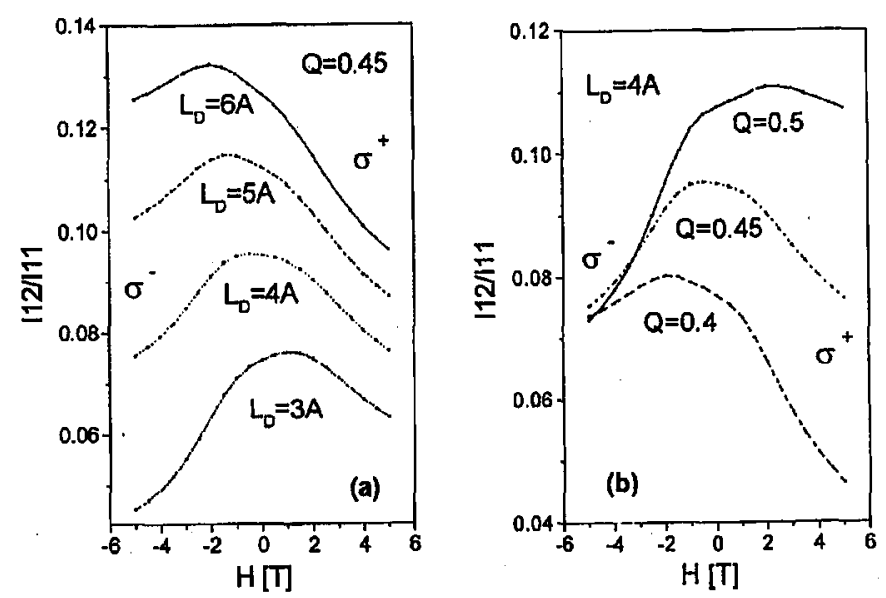

Fig. 3. Magnetic field dependence of the ratio of the additional and main lines intensities $(I 12 / I 11)$ calculated for a single $\mathrm{QW}$ with the interface function $F(t)$ in the form (2), with the parameter $s=1$ for different values of $L_{\mathrm{D}}$ (a) and valence band offset (b) parameters. Positive values of the magnetic field $H$ correspond to $=\sigma^{+}$polarization, negative ones to $\sigma^{-}$. 

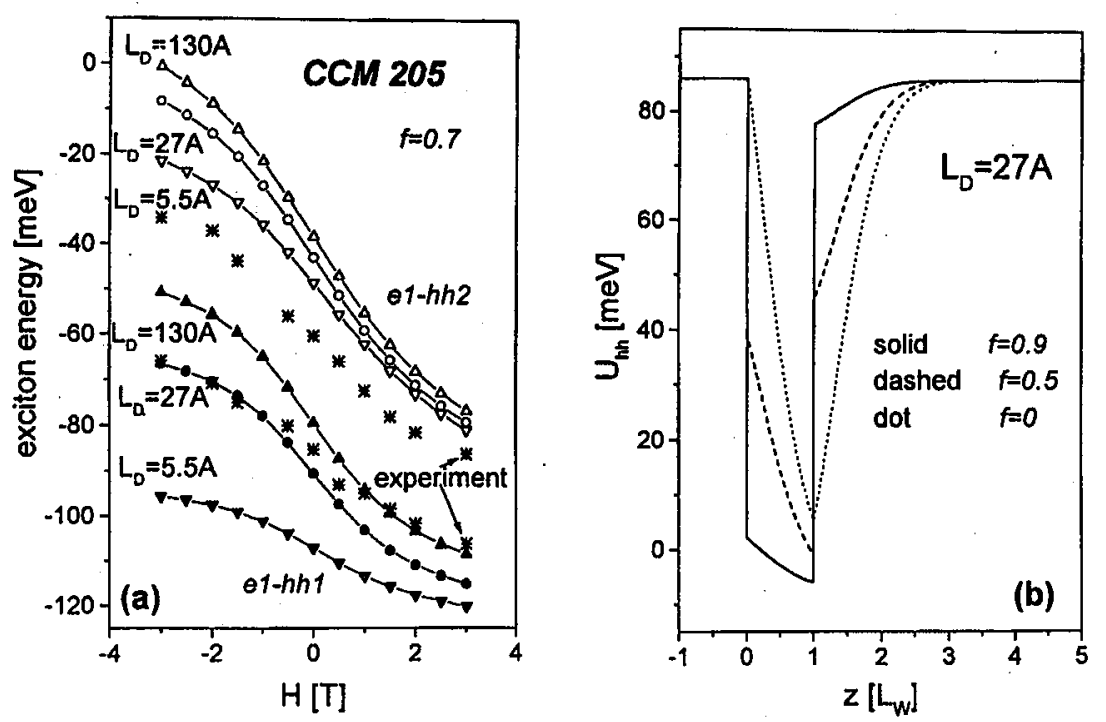

Fig. 4. Calculated values of the e1-hh1 (main line) and e1-hh2 (additional line) transitions energies (a) for CCM-205 sample using the "two-stage" interface function (4) plotted as a function of the magnetic field. Experimental points (stars) are plotted for comparison. Positive values of the magnetic field $H$ correspond to $\sigma^{+}$polarization, negative ones to $\sigma^{-}$. Heavy hole potential profile (b) in zero-magnetic field plotted for different values of the parameter $f$.

Schrödinger equations for electrons and heavy holes motion in $z$-direction numerically using the "shooting" method. We determined the wave functions and eigenenergies for electron and heavy hole ground states (e1 and hh1, respectively) and for heavy hole first excited confined state hh2. The relation of the intensities of the main and additional lines was calculated as the ratio of squares of envelope function overlap integrals for electron and hole associated with the additional and main line transitions, i.e., for e1-hh2 for the additional line and e1-hh1 for the main line. As far as the present work does not raise the question of full quantitative explanation of the experiment we use for exciton binding energies and strain effects description only very approximated values of the parameters.

The calculations have been performed for different types of the interface function $F(t)$ with different values of the parameters $L_{\mathrm{D}}, s, f$, for different structure parameters and different values of the valence band offset parameter $Q$. Some results of these calculations are given in Fig. $3 \mathrm{a}$, b. In Fig. 4a, b we compare the results of the calculations with the experimental results obtained for the sample CCM-205. Zero energy in Figs. 4a, 5b, 6 corresponds to the energy position of the barrier exciton line in zero magnetic field.

In the case of SL (CCM-207) we approximate the miniband effects by consideration of the tunnel-split of quantum confined states in the double quantum well (DQW) structure with the barrier width $L_{\mathrm{b}}$. We assume that the lower tunnelling state in the case of DQW corresponds to the state at the bottom of mini- 

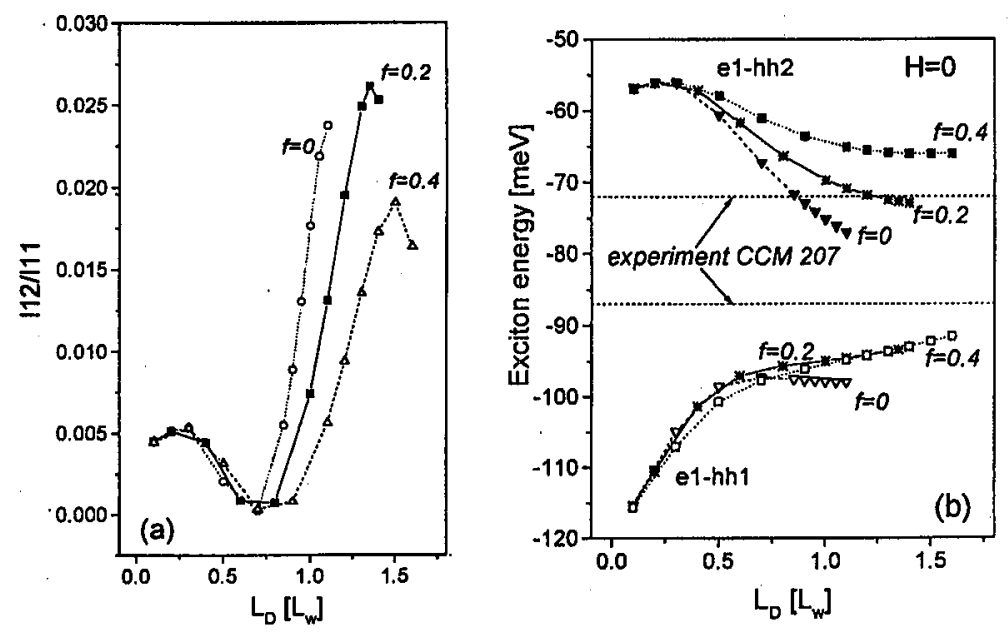

Fig. 5. Ratio of the additional and main lines intensities (II2/I11) (a) and energies of the e1-hh1 (main line) and e1-hh2 (additional line) transitions (b) calculated for double QW case in zero-magnetic field with the interface function $F(t)$ in the form (4) for different values of the parameters $L_{\mathrm{D} 2}$ and $f$.

band in the case of SL, i.e., to the state with the zero value of the miniband wave vector. In the case of DQW we approximate the Mn distribution in $z$-direction by the following function:

$$
\begin{aligned}
x(z) & =x_{\mathrm{B}}\left[F(z-0)+1-F\left(z-L_{\mathrm{w}}\right)\right. \\
& \left.+F\left(z-L_{\mathrm{w}}-L_{\mathrm{b}}\right)+1-F\left(z-2 L_{\mathrm{w}}-L_{\mathrm{b}}\right)\right] .
\end{aligned}
$$

The calculations were performed both for the "one-stage" interface function $F(t)$ in the form (2) or (3) and for the "two-stage" function in the form (4) or (5) in the wide range of the parameters $L_{\mathrm{D}}, s, f$, etc.

In Fig. 5a, b we present the results of the calculations of the relative intensity of the e1-hh2 optical transition and transition energies for different parameters of the interface function $F(t)$. Figure 6 shows the magnetic field dependence of the transition energies in comparison with the experimental points for CCM-207 sample. The positive and negative values of the magnetic field $H$ correspond to $\sigma^{+}$and $\sigma^{-}$components, respectively.

The performed analysis shows:

(i) The calculated values of the energy positions of main (e1-hh1) and of additional (e1-hh2) lines and the ratio of their intensities for $\sigma^{+}$and $\sigma^{-}$GSS components are in principal agreement with those from the experiment for the CCM-205 and CCM-207 structures. The $L_{\mathrm{D}} / L_{\mathrm{w}}$ ratio should be taken as relatively large (up to $\cong 1$ ) for the best agreement.

Note that the calculated GSS value is a bit greater than the experimental one. We suppose that for better agreement with the experiment it is useful to take into account the Coulomb interaction in a similar way as in Ref. [18]. 


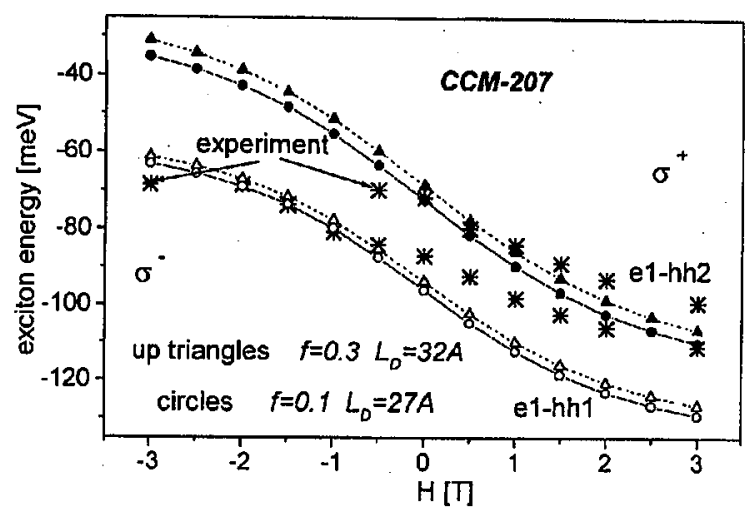

Fig. 6. Magnetic field dependence of the e1-hh1 (main line) and e1-hh2 (additional line) transitions energies for CCM-207 sample calculated using the "two-stage" interface function (4). Experimental points (stars) are plotted for comparison. Positive values of the magnetic field $H$ correspond to $\sigma^{+}$polarization, negative ones to $\sigma^{-}$.

(ii) The results of calculations are very sensitive not only to interface intermixing length parameter $L_{\mathrm{D}}$, but to the type of interface function $F(t)$ contrary to the GSS "paramagnetic enhancement" effect $[16,17]$, where the $L_{\mathrm{D}}$ value is important only. The important characteristic of the interface profile is the magnetic field dependence of the ratio of the additional and main lines intensities. Using different sets of the interface function $F(t)$ parameters it is possible to obtain in calculations the increasing of this ratio either for $\sigma^{+}$components or for $\sigma^{-}$ones. It gives us an additional possibility to control the model validity by compare the calculated dependence of this ratio with the experimental data.

This situation gives a possibility to use the observation and analysis of additional lines in QW spectra to obtain the information about details of interface profiles for different growth technologies.

\subsection{Conclusion}

Based on the computer simulations of the main and additional lines energy positions and relative intensities we conclude, that the additional line in $\mathrm{QW}$ spectra might be explained in terms of $\mathrm{hh} 2 \rightarrow \mathrm{e} 1$ transitions, which ceases to be forbidden in the presence of technologically caused asymmetry of QW potential profile. The sabre-like profile of $\mathrm{Mn}$ concentration distribution was used in the vicinity of the $\mathrm{QW}$ interfaces. It was shown that the asymmetry parameter depends on the peculiarities of the growth technique. In particular, it is essentially larger for our samples grown by the laser ablation method than for MBE grown ones.

This investigation was partly supported by INTAS grant \# 93-3657 exp. and by grant of State Fundamental Research Foundation of Ukraine \# F4/346-97. 


\section{References}

[1] S.P. Shubin, S.V. Vonsovskij, Proc. R. Soc. Lond. A, Math. Phys. Sci. 145, 159 (1934).

[2] S.V. Vonsovskij, Zh. Eksp. Teor. Fiz. 16, 981 (1946).

[3] S.V. Vonsovskij, E.A. Turov, Zh. Eksp. Teor. Fiz. 24, 419 (1953).

[4] S.V. Vonsovskij, Magnetizm, Nauka, Moscow 1971 (in Russian).

[5] J. Kondo, J. Appl. Phys. 37, 1177 (1966).

[6] D. Pines, J. Bardin, Ch. Slichter, Phys. Rev. 106, 489 (1957).

[7] Z. Methfessel, D.C. Mattis, Magnetic Semiconductors, in: Handbuch der Physik, Ed. S. Flügge, Vol. 18/1, Springer Verlag, Berlin 1968, p. 389.

[8] E.L. Nagaev, Physics of Magnetic Semiconductors, Mir, Moscow 1972, translated from Russian.

[9] M.F. Dejgen, V.Ya. Bratus', B.E. Vugmeister, I.M. Zaritskij, A.A. Zolotukhin, A.A. Konchits, L.S. Milevskij, Zh. Exp. Teor. Fiz. 69, 2110 (1975).

[10] A.V. Komarov, S.M. Ryabchenko, I.I. Zheru, R.D. Ivanchuk, O.V. Terletskij, in: 5th Symposium on Spectroscopy of Crystals Activated by Fe-Group Ions, Kazan 1976, Book of Abstracts, p. 88.

[11] A.V. Komarov, S.M. Ryabchenko, I.I. Zheru, R.D. Ivanchuk, O.V. Terletskij, $Z h$. Exp. Teor. Fis. 73, 608 (1977).

[12] E.A. Pashitskij, S.M. Ryabchenko, Fiz. Tverd. Tela 21, 545 (1979).

[13] Yu.G. Semenov, B.D. Shanina, Phys. Status Solidi B 104, 631 (1981).

[14] S.M. Ryabchenko, Yu.G. Semenov, Zh. Exp. Teor. Fiz. 84, 1419 (1983); Fiz. Tverd. Tela 27, 3347 (1984).

[15] S.M. Ryabchenko, Yu.G. Semenov, O.V. Terletskij, Phys. Status Solidi B 144, 661 (1987).

[16] J.A. Gaj, W. Grieshaber, C. Bodin-Deshays, J. Cilbert, G. Feuillet, Y. Merle d'Aubigné, A. Wasiela, Phys. Rev. B 50, 5512 (1994).

[17] W. Grieshaber, A. Haury, J. Cibert, Y. Merle d'Aubigné, A. Wasiela, J.A. Gaj, Phys. Rev. B 53, 4891 (1996).

[18] A. Ribayrol, D. Coquillat, J.P. Lascaray, A.V. Kavokin, D.E. Ashenford, Phys. Rev. $B$ 51, 7882 (1995).

[19] M. Kutrowski, T. Wojtowicz, G. Cywiński, G. Karczewski, E. Janik, E. Dynowska, J. Kossut, Acta Phys. Pol. A 92, 887 (1997).

[20] A.P. Roth, R. Benzaquen, P. Finnie, P.D. Berger, J.J. Dubowski, Proc. SPIE 2045, 322 (1994). 